\title{
The Web is Big Business
}

\author{
Rizwan Mehmood ${ }^{1}$ and Petra Korica-Pehserl ${ }^{2}$ \\ ${ }^{1}$ Centre for Distributed and Semantic Computing, Mohammad Ali Jinnah University, Islamabad, Pakistan \\ ${ }^{2}$ Department of Computer Engineering, University of Belgrade, Serbia
}

\begin{abstract}
As has been explained in the paper "A Survey of Communications and Collaborative Web Technologies" in this special issue the web started as a more or less one-way information system, allowing engineers at CERN to share new results in physics world-wide easily. As it developed, it became more and more a two-way (or more precisely a multi-way) communication tool, allowing to collaboratively work in many ways. This has, as it has been described in the aforementioned paper, not just changed how information is created and provided easy ways of communication, but has also allowed the growth of social networks as first tools to tie together the power of brains, changing how we work, think, do research, form new circles of people we are interested in. This on its own would have been a major change in how society works, yet it turned out soon that the technologies available would allow to set up completely new business models, and threaten some existing ones. Among the hundreds of million applications found on the web today, many set up to make money, or at least pay for themselves by providing excellent ways of advertisements. There is no person who could know all the applications available on the web, and there is no way to even list a tiny percentage of them. However, what we are trying in this paper is to classify applications and discuss their commercial or societal impact.
\end{abstract}

Keywords: emerging technologies, web applications

\section{Introduction}

The aim of this paper is to try to define a number of categories of applications now visible on the web, and to give a few examples for each category. We further try to point out developments that will affect the influence of the web in many ways.

Almost all applications on the web are either conceived as businesses to make some money, or justify their existence by generating enough income to maintain them from revenues generated by advertisements, often in conjunction with the "real world". A small percentage is maintained by idealists who participate because they believe in the importance of their work, in this case often supported by donations or sponsors. The most famous example is certainly Wikipedia [1] with European smaller brothers like the Austria-Forum [2] and the Serbia-Forum [3]. Particularly [1] has been and is based on the "Wisdom of the Crowds" [4], despite early warnings in [5]. Talking to companies, advising on how to make business on the web, we have often heard of the 1-2-3-4-model: there are "only 4 ways": 1 . Collect Donations 2 . Sell Advertising 3. Sell Your Own Product 4. Sell Someone Else's Product. There are many similarly superficial advices as e.g. found in [7] and totally different models like [18].

Our paper is divided into two parts: the first one is a first (and certainly not an exhaustive) classification of web applications that does not follow previous models. The second one is a look at some figures and developments, a good part based on [6] and other not quite so substantial resources.

\section{Categories of Applications}

\subsection{Web Applications Mainly Dealing with Items Not Available on the Web}

This category comprises all sites that offer goods for sale, like Amazon, eBay, Alibaba, all sites that allow the booking of hotels, events, transportation, etc.

Notice that such applications are certainly not "small fry": Amazon Web Services' revenues are on track to hit the $\$ 1$ billion mark in the fourth quarter 2014. If they do, AWS will have generated an estimated $\$ 3.2$ billion in revenues 
for parent company Amazon.com - a striking accomplishment for a business still in the "other" category on the balance sheet [8].

Or consider Alibaba that just raised over \$25 billion by going public. Some might say this is a sign that we are approaching another Internet bubble. After all, since trading started the shares have lost some $10 \%$ of their value within days [9] and the company got much criticism for the poor translation into other languages. In summary, there are thousands of companies of all sizes that peddle their merchandize or products of others via the net. And if sales as such are not always completely satisfying, the advertisement effect has to be added into the total configuration.

\subsection{Web Applications Mainly Offering Items that Are on the Web}

This category includes all companies selling music or video via the web, with Apple's iTunes store one of the early and still successful sources. Note also that a large majority of companies offer a trimmed down or trial version for free (like encyclopedias Brockhaus and Britannica, [10] and [11]), but charge for the full (or beyond trial version). Many sites offering sophisticated (often multi-person games) belong here. It is also fair to count into this category also some players that were big from the start, yet nobody really wants to write about them. We are talking here about gaming/betting/casino applications (where lack of control has led to serious ripoffs) and about pornographic sites. It is worth to ponder why some 30.000 Internet users are viewing pornography every second, or why despite a tremendous amount of free material, some 90 billion dollars (10 million per hour) are spent on watching pornography, if we believe sources like [12].

\subsection{Web Applications Dealing with Support of Activities on the Web}

This category includes all organisations that offer web hosting, space on the cloud, software of any kind, hardware, software, but also I/O devices, the booming market of apps (much more important than advertisement in the smartphone market, see [6]) and, yes, of course, all search engines. The two really large ones known in the Western world Google and Bing reap huge profits through banners and ads in conjunction with agencies trying to optimize sites, or making better placement of search results possible for payment. And some, like Wolfram Alpha, work on the basic vs. full model.

\subsection{Picture Applications}

In the picture area the community, the crowd, plays a particularly strong role. Per day some 2 billion pictures are uploaded on Snapchat, Flickr, Facebook, Instagram, WhatsApp (which during the time of writing was bought by Facebook for over 10 billion \$). But we have also pictures on Wikipedia or Wikicommons, and in zillions of other picture collections [13] or [14]. In some cases pictures can be used freely, like many pictures from NASA or the CIA Factbook, in other cases pictures have to be purchased in every case, in still other modes pictures can be used under some kind of Creative Commons or GNU licences, or like in [14] many low resolution pictures can be used freely for non-profit use, but higher resolution versions have to purchased. Many services allow the sharing of pictures in well-defined groups.

\subsection{Communication and Video Applications}

Due to its architecture, web is an ideal platform for communication via voice and video. The way this is implemented is using Internet Protocol (IP) telephony based technologies. IP telephony is a general term for the technologies that use the Internet Protocol's packet-switched connections to exchange voice, fax, and other forms of information that have traditionally been carried over the dedicated circuit-switched connections of the public switched telephone network (PSTN). Using the Internet, calls travel as packets of data on shared lines, avoiding the tolls of the PSTN [41]. This is especially important for long-distance calls and video conferencing. Skype [42] was one of the first to popularize this. Google has its service for group calls and video conferencing called Google hangout [47]. Microsoft's Lync [48] and Cisco [49] are offering these services for business customers. The monetization of those services is mostly done through the licencing of the business version or offering prime services as add-on to a free consumer version like both Google hangouts and 
Skype charge for calls to mobiles and landlines worldwide on a subscription basis.

Given that we can easily transmit voice and video, the next step is Internet Protocol television (IPTV). IPTV is a system through which television services are delivered using the Internet protocol suite over a packet-switched network such as a LAN or the Internet [50] enabling a new generation of content consumption. A popular application of that is video on demand which enables users to instantly watch any video that they want when they want, assuming that the provider has that video in their catalogue. Netflix [51], a web service that instantly streams TV shows \& movies over the Internet, was one of the first to popularize this. Amazon offers a similar service called Amazon Instant Video [52]. Monetization in these cases is done by monthly subscription fee. A possible future monetization channel is through targeted ads by creating patterns out of the collection of viewer's data and habits through big data technologies, see [53] for example.

Another very popular service in this category is YouTube, which is the most popular video service on the Internet with over 1 billion unique users visits each month from 61 countries [45]. This is a great engine for making money from advertising and therefore YouTube uses Google's AdWords for advertising. Advertisers can play their video ads before the video itself, besides videos being played or in search results. YouTube pays for the video advertisements that user has seen in full. Additionally it is possible to buy a channel sponsorship which consists of display, overlay and pre-roll ads. This offers a possibiltiy for targeted marketing as it can specially target viewers based on their preferences. See [43] and [44] for details.

\subsection{Geoservices}

Recent years have seen remarkable growth in geo services due to advances in remote sensing, satellite communication and GPS technology. GIS industry is making progress [22]. City level strategic planning and decision making is now benefitted from many open source GIS systems like QGIS, PostGIS which are available free of cost. The list of open source GIS is quite long and can be seen in [23].

Spatial databases have made spatial analysis much easier. Almost all database management system vendors like SQLServer, Oracle, and PostgreSQL are now eyeing at improving spatial features and enhancing their spatial querying capabilities. These databases support desktop GIS as they provide efficient way of storing and manipulating geographic data which was previously stored in the form of shape(.shp) files on hard disk.

Web GIS is another emergent area. Web mapping frame-works like Map fish, Geo server and freely available mapping libraries such as Openlayers, Leaflet [24], [25] are empowering desktop GIS researchers to use Web as a medium for geographic analysis.

The OpenStreetMap (OSM) [26] is a freely editable map. It was started by Steve Coast in 2004.

Unlike Google maps, Open street map data is freely available and it provides very valuable information within city and country levels. Osmosis [27] is a command line Java application for processing OSM data. An osmosis command which is used to extract schools in Austria from openstreetmap is:

$$
C: \backslash>\text { osmosis - rbf austria.osm.pbf - nkvkeyValueList = "amenity.school" }- \text { wxschool.osm }
$$

The OSM data has the advantage that once extracted, it is precisely plotted on the map because of the geographic location attribute that is stored with every single object in OSM map. The map of schools in Austria is shown in Figure 1. It gives a quick impression of the density of schools in certain areas and it helps in strategic decisions regarding opening new schools. Considering online routing systems, Google maps,
Virtual Earth and Nokia HERE Maps are some of the main players.

In connection with providing geo services, a noteworthy effort is GeoNames. It is a geographical database that contains data about all countries and it has over eight million place names. It provides web services to access the required data. 


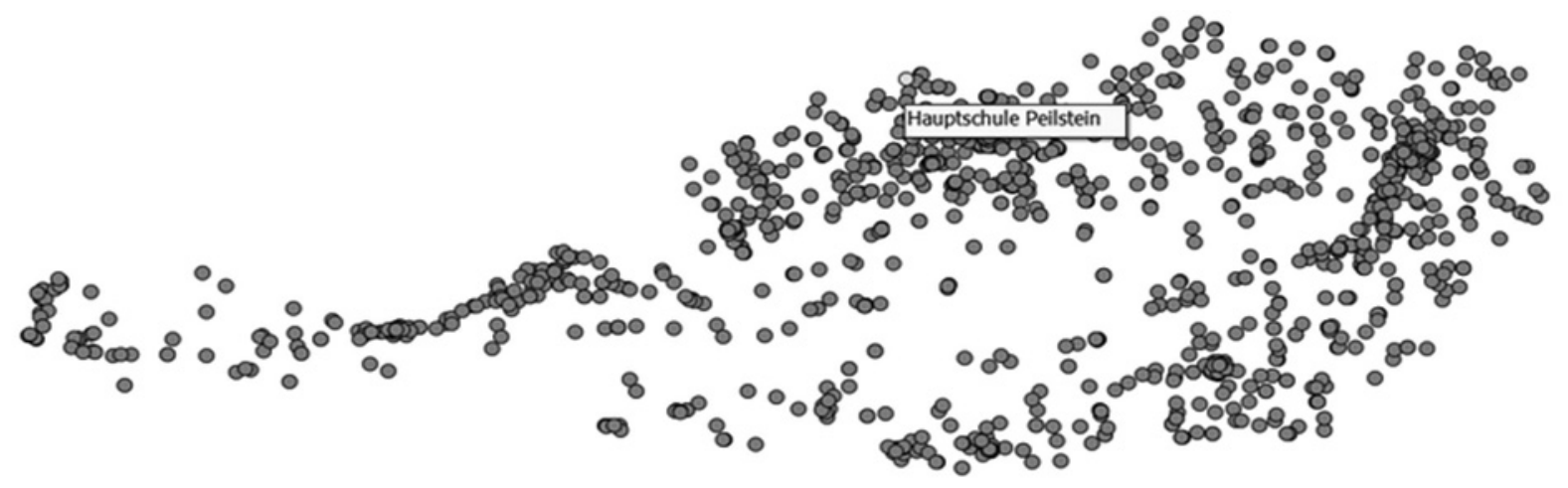

Figure 1. Map displaying schools in Austria; Data taken from OSM [26].

A distinguished online collection on geography is "The CIA world fact book". It covers different aspects of geography. The data can be downloaded from CIA world fact book freely. It provides an archive of geographic information for the last 10 decades, which can be used for statistical inference. It is updated biannually to provide up to date information.

It is worth to mention here "Geography of the world", a unique project on its own where data from multiple data sources are merged together. The major emphasis behind this project is to provide reliable information to people; see [17]. The wiki page of Austria is shown in Figure 2.
Ranging from culture, maps, government up to geography, the users can find almost everything they want to know about a particular country. We take care of minute details related to every country. For instance, flag and emblems of a particular country are displayed on the respective wiki page of that country. The icons lead to sub wiki pages where users can find corresponding information. The main idea of this effort is not so much to collect data from different sources, but to assure correctness as much as possible by comparing data from up to then different geographic data bases and geographic search engine.
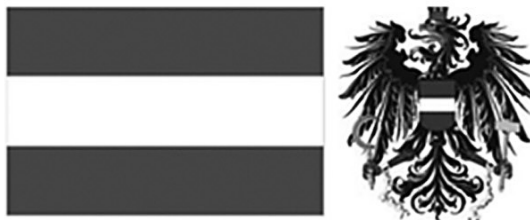

Once the center of power for the large Austro-Hungarian Empire, Austria was reduced to a small republic after its defeat in World War I. Following annexation by Nazi Germany in 1938 and subsequent occupation by the status remained unclear for a decade. A State Treaty signed in 1955 ended the occupation, recognized Austria's independence, and forbade unification with Germany. A constitutional law that same year declared the country's "perpetual neutrality" as a condition for Soviet military withdrawal. The Soviet Union's collapse in 1991 and Austria's entry into the European Union in 1995 have altered the meaning of this neutrality. A prosperous, democratic country, Austria entered the EU Economic and Monetary Union in 1999.

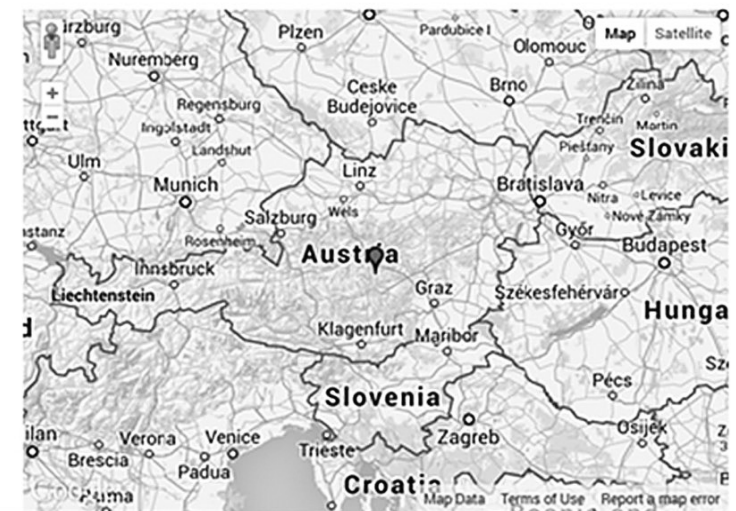

Geography

Figure 2. The wiki page of Austria under Geography of the World Project; picture taken from [2]. 


\subsection{Virtual Presentations and Digital Libraries}

There are numerous websites that offer virtual visits to cities, places of interest, museums and the like. One of the largest efforts world-wide is probably Europeana [28] with some 40 million documents from over 2.000 participating museums. As impressive as the project is, it has one major drawback: it acts only as portal, i.e. as one visits a different museum, the interface and even the language can change completely. Another more homogeneous example is the Smithsonian in Washington [15] that started as a nicely done virtual tour, but since many visitors asked questions about just anything, the museum decided to collect the questions and answers in a separate collection that has now reached the dimension of a substantial encyclopaedia, part of it allowing interactive exploration through games! Both Europeana and Smithonian are also a bridge to virtual libraries. A number of such very large efforts have attracted much attention, like the Open Library [29] with over

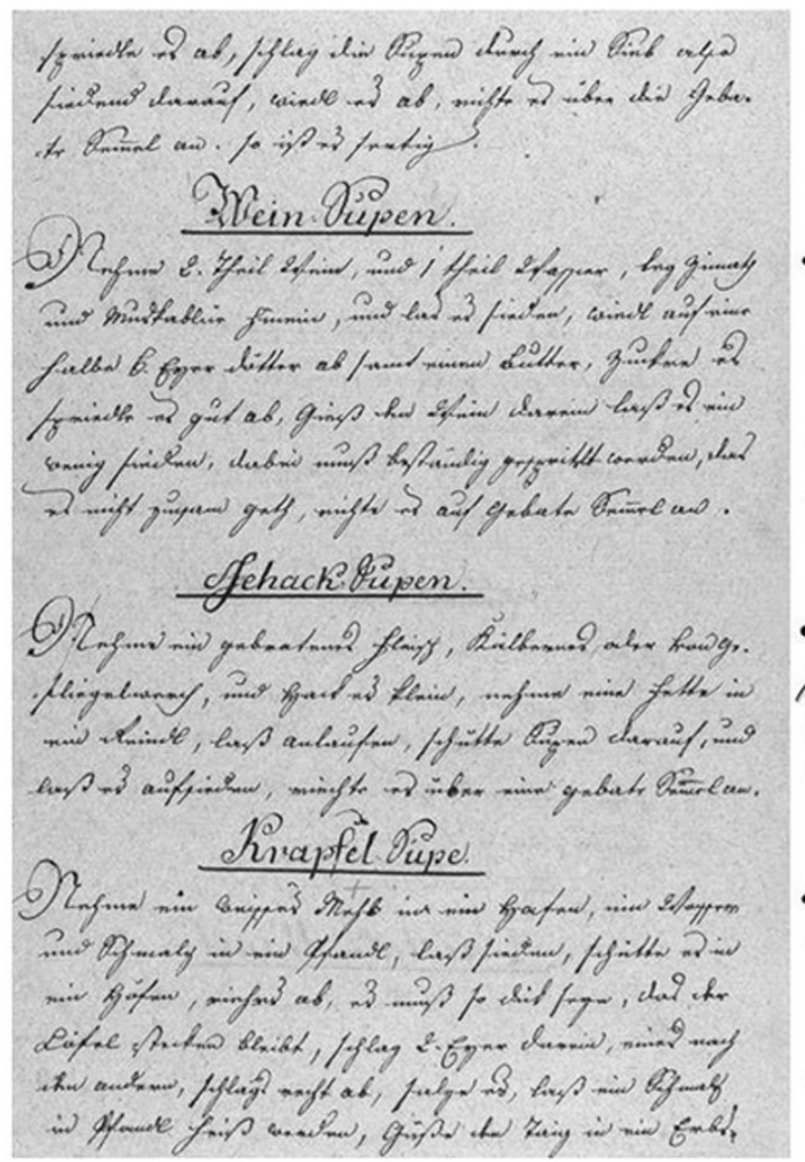

one million classical books accessible without charge, and a "borrowing service" for some 100.000 more recent books: a book can only be borrowed if nobody else is currently using it, a rather severe restriction that one also encounters in many other libraries that offer books in some electronic form. Google Books [30] combines offering books for free or buying them, often with the possibility of first being able to read a bit. Searching for digital libraries on the web, one finds over 100 projects. Often books can be read as pdf File, or with specialized e-Book readers. One small collection of books [31] deserves particular attention since all is free and the book viewer offers unusual features, like displaying side by side the same page in different fonts or languages. An example found of a page in an old cookbook from [32] is shown in Figure 3: one can see a page of the handwritten book (hardly decipherable) on the left, but to the right of it the same material in a transcribed form. This feature could also be used to show e.g. the page of a book in English on the left, and the same page in German on the right.

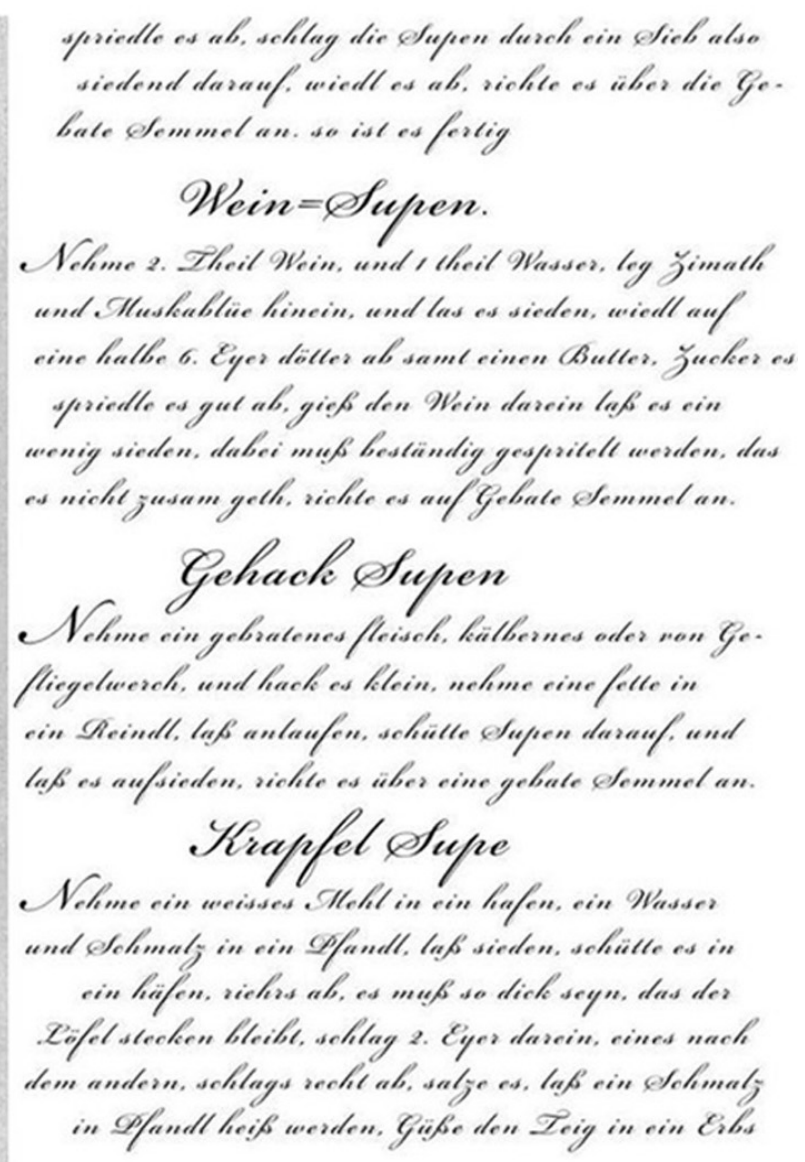

Figure 3. Same page; left: handwriting, right: transcription. 


\subsection{Advertising Services}

One of the most widely used instruments for advertising on the web is Google Adwords, an online advertising service that places advertising copy at the top, bottom, or beside the list of search results Google displays for a particular search query. The choice and placement of the ads is based in part on a proprietary determination of the relevance of the search query to the advertising copy. AdWords has evolved into Google's main source of revenue. Google's total advertising revenues were $\$ 42.5$ billion in 2012 [21].

However, Internet businesses are still making use and are expanding many traditional models. For instance, auctions are being used by many market leaders and startups that allow users to bid for unique price value for each product item like in eBay[19]. A taxonomy of business models, different from what we used in Section 2, is found in [18], dividing applications categories into Brokerage, Advertising, Infomediary, Merchant, Manufacturer, Affiliate, Community, Subscription and Utility. A broadcasting style of marketing still has strong appeal, with an ability to reach millions of potential customers through a variety of means. Advertisements are producing gigantic profits for many players on the web. By employing a "Freemium model", information is made available free to users to "whet the appetite". Targeted advertisements are placed strategically, either according to location of users, or the natural language associations for matching products to users.

Advertisements are directed towards users, who are pursuing particular information, at some point before or during the information provisioning. Often approached transparently, ads move into the user space and there are situations where these advertisement tend to be intrusive.

Data is collected extensively and includes demographic information for a multifaceted profiling of users to enhance the possibilities of clicks on the adverts. Google has pioneered content targeted advertisement, and are able to integrate all their services to gain access to targeted audience who are likely buyers of products or services. Users may also opt to consent to placement of advertisements within their con- tent spaces or websites to share profits based on numbers of users.

In 2013 the advertising revenue came close to 120 billion [6]. Contrary to what we will see in Section 3, the big advertisement market is still with PCs, less than $10 \%$ from mobile devices.

\subsection{Web Applications Dealing with Connecting People over the Web}

This category includes all applications (web or mobile) and/or web sites that offer possibility to meet, collaborate with or hire people using web. Examples of such sites are online-dating sites like OKCupid [33] or Tinder [34], to find romance/friends, online job ad sites like Monster [36] or Amazon Mechanical Turk [35] for crowdsourcing the tasks. Other sites that fall into this category are applications that enable you to connect and talk to strangers - see [36] for iOS platform and [37] for Android platform, for example, and business apps to meet people and potential contacts/partners/clients at the conferences and business events like [38]. For the completeness of this paragraph we must not forget older sites like LinkedIn [39] or XING [40].

\subsection{Notes}

We have omitted many other offerings for both space reasons or because they do not neatly fit into any of the above categories. This includes consulting services (Telemedicine), rating services, translation services etc. We have not mentioned the large number of e-Learning applications since they are treated in another part of this special issue. Last but not least, we have not mentioned the danger of violent games, cybercrimes (steadily increasing) and other potential dangers of the web since these topics are considered in detail in [16].

\section{Some Trends and Figures}

In what follows we rely heavily on [6], [18] and $[20]$ and similar reports. 


\subsection{Development Trends Overall}

The year to year growth on the Internet has slowed to less than $10 \%$, and even more so in developed countries. Smartphones are still growing at over $20 \%$, and mobile traffic over $80 \%$ with video as the main driver. Tablets are growing faster than laptops, and PC sales are slightly dropping. Mobile phones have by now reached almost the same penetration as TV. Mobile Internet use has about doubled (world-wide fairly evenly) between 2013 and 2014 .

\subsection{Result of the Internet Bubble and Potential "Killer" Applications}

The technology company value market reached its peak on March 10, 2000, then dropped by $50 \%$. Recovery has happened since then (both in value and IPOs), but is still more than $35 \%$ below the highest point.

e-Learning that has not taken off for 60 years seems to have a real chance now: not only is education becoming more and more expensive, it is also considered more desirable, and technologies (including collaboration technologies) could lead to a break through. Current growth figures are encouraging. The use of online courses (Khan Academy You Tube Chanel, iTunes U Open University, and Coursera course use has about doubled in a year). Duolingo with 25 million users is most popular in Europe, Coursera with 7 million users in North America.

What has just been said for eLearning may also apply to the medical sector. IT technologies and telemedicine seems to soften the problem of rising medical costs.

Music distribution may not be a killer application yet physical music sales are constantly dropping, while music streams increase by some $30 \%$ per year.

The effect of Cryptocurrencies (Bitcoin, Coinbase, Multibit, Blockchain) is not yet clear. However, 5 million bitcoin wallets (an 8 fold increase in a year) makes it clear that there is extraordinary interest in this area.

\subsection{Development of Communication Services}

Facebook is no.1, but broadcasting fewer messages to a larger audience (and is clearly trying to change this by recently acquiring WhatsApp). Other (surprisingly mainly national) services are growing fast: WhatsApp (USA) grew over $150 \%$ in a year, Line (Japan) by $500 \%$ with $\$ 388$ million revenue, also Tencent WeChat (China), Kakao Talk (Korea) and Viber (Israel) have strong growth. The pattern how people seem to meet (if US can be taken as valid also elsewhere) is fascinating: There are $70.000 \mathrm{bar} /$ nightclubs in the USA were people can meet, but the service Tinder produced 11 million "It's a match" per day, a 21 fold increase in a year.

\subsection{Sensors, New I/O Devices, Data Mining and Analytic Tools}

The number of sensors in smartphones is increasing all the time; the iPhone (2007) had three sensors (accelerometer, proximity, ambient light), the Galaxy S5 (20143) has ten: Gryo, fingerprint, barometer, control of hull, ambient light, gesture, heart rate, accelerometer, proximity, compass: the use of MEMS (microelectromechanical systems, i.e. sensors and activators) has been increasing more than $30 \%$ per year since 2007 !

This and new display technologies (like headup-display like Google glasses), 3D picture and video, high resolution large screens, speech recognition and speech generation from text together with data mining for Personalisation (Netflix), Data analysis (Ayasdi) also for medical applications (personalized medicine) are strong driving forces.

\subsection{Convergence of TV Screens and End of TV}

New TV screens with remote control that turn their TV screen into a display screen for $\mathrm{PC} /$ smartphone etc. are emerging. Linear TV channels will be more and more replaced by on-Demand video. The 9 YouTube channels 
(Music, Gaming, Sports, News, Popular, Spotlight, Movies, TV Shows, Education) all have growth rates of over $100 \%$ per year, Spotlight over $300 \%$. However, looking at a YouTube channel will not stop there, but may well lead to chats (Google hangouts) with persons you would never talk to/meet otherwise. On the horizon are videos (like now in gaming) where the spectator becomes part of the action.

\subsection{Global Players}

In terms of the market value, the four US companies Apple (529), Google (377), Facebook (157) and Amazon (144) are still ahead (numbers are billion \$), but Chinese Tencent (132) is not far behind and Chinese Alibaba is catching up. Note, however, that financial analysts have spilled oceans of ink trying to compare Amazon to Alibaba. Both are in the e-commerce area, but have different business models, with Alibaba just collecting fees for providing the electronic platform, while Amazon actually owns and ships physical goods.

Independent of this, it seems likely that Chinapartially due to its gigantic smartphone marketwill soon be a mobile commerce innovation leader.

\section{Conclusion}

In this paper we have tried to show how closely the commercial/business market is interwoven with technologies of communication and collaboration on the Web.

However, let us not forget that the Internet is more than the web. As far as the basic infrastructure of the Internet is concerned, most of the revenue is still coming from voice as Odlyzko shows. He presents some statistics in [20] proving his claim.

\section{Acknowledgments}

We want to thank Andrew Odlyzko from the University of Minnesota for helping us find concrete figures and examples in the vast ocean of the web.

\section{References}

[1] Wikipedia, http://www.wikipedia.org/

[2] Austria-Forum, austria-forum.org

[3] Serbia Forum, www.serbia-forum.org

[4] J. SuROwIECKI, The wisdom of Crowds. Anchor Books, 2005.

[5] A.KeEN, The cult of the amateur. Double Day, 2007.

[6] M. MEEKER, Internet Trends 2014. http://www.kpcb.com/Internet-trends

[7] http://webmarketingtoday.com/articles/ wilson-ad-revenue-4/

[8] http://www.informationweek.com/cloud/in frastructure-as-a-service/amazon-web-se rvices-revenue-new-details/d/d-id/11120 $68 ?$

[9] http://www.finanzen.at/aktien/AlibabaAktie

[10] http://www.britannica.com/

[11] www.brockhaus-enzyklopaedie.de/

[12] http://www.ministryoftruth.me.uk/wpcontent/uploads/2014/03/IFR2013.pdf

[13] http://www . imagno.com

[14] http://digitalgallery.nypl.org/nypldi gital/index.cfm

[15] The Smithsonian http://www.si.edu/

[16] H. Maurer, R. Mehmood, P. KoricA, How Dangerous is the Web for Creative Work. CIT 2 (2013), pp. 59-70.

[17] N. KulathuramaiYer, H. MAURER, R. MEHMOOD, On the reliability of Web pages. To appear in JUCS.

[18] M. RAPPA, Business Models on the Web, (2010). http://digitalenterprise.org/mod els/models.html

[19] eBay http: //www.ebay.com/

[20] A. ODLYZKO, will smart pricing finally take off? In: "Smart Pricing" (S. SEN, C. JOE-WonG, S. HA, M. ChiAng, Eds.) Wiley (2004), 3-33, reprint at http://www.dtc.umn.edu/ odlyzko/doc/sm art.pricing.pdf

[21] http://investor.google.com/financial/ta bles.html

[22] D. DANKo, W. KRESSE, Springer Handbook of Geographic Information, 2012.

[23] Open Source GIS, http : //opensourcegis.org/

[24] Openlayers, http://openlayers.org/ 
[25] Leaflet, http://leafletjs.com/

[26] http://www . openstreetmap.org/\#map=9/47 $.0701 / 15.0952 \&$ layers $=Q$

[27] http://wiki.openstreetmap.org/wiki/Osmo sis

[28] http://www.europeana.eu/

[29] https://openlibrary.org/

[30] http://books.google.com/

[31] http://austria-forum.org/af/Web_Books

[32] http://austria-forum.org/wbg3/?handschr iftlich00de1818kfu

[33] https://www.okcupid.com/

[34] http://www.gotinder.com/

[35] http://aws.amazon.com/mturk/

[36] http://appcrawlr.com/ios-apps/best-apps -talk-to-strangers

[37] http://appcrawlr.com/android-apps/bestapps-talk-to-strangers

[38] http://www.topi.com/

[39] http://www.linkedin.com

[40] http://www.xing.com

[41] http://searchunifiedcommunications.tech target.com/definition/IP-telephony

[42] http://www.skype.com

[43] http://www.youtube.com/yt/advertise/ind ex.html

[44] http://thenextweb.com/google/2012/04/02 /youtube-rolls-out-channel-sponsorshipad-model-reportedly-charging-up-to-62mfor-exclusivity/

[45] https://www .youtube.com/yt/press/en-GB/ statistics.html

[46] http://www.monster.com

[47] https://www.google.com/+/learnmore/han gouts/

[48] http://products.office.com/en-us/lync/ lync-2013-video-conferencing-meeting-s oftware

[49] http://www.cisco.com/c/en/us/products/ conferencing/index.html

[50] http://en.wikipedia.org/wiki/IPTV

[51] https://www.netflix.com/global

[52] http://www.amazon.co.uk/Instant-Video/b $?$ ie $=$ UTF $8 \&$ node $=3010085031$

[53] http: //www.salon.com/2013/02/01/how_net flix_is_turning_viewers_into_puppets/
Received: October, 2014 Accepted: October, 2014

Contact addresses:

Rizwan Mehmood

Centre for Distributed and Semantic Computing Mohammad Ali Jinnah University

Islamabad

Pakistan

e-mail: rizwanmehmood@hotmail.com

Petra Korica-Pehserl

Department of Computer Engineering

University of Belgrade

Serbia

e-mail: pekorica@outlook.com

RIZWAN MEHMOOD started his professional career as a Software Engineer. He joined Makabu (a leading software company in Pakistan) in 2005 and was involved in the development and design of many desktop and web applications. He provided his services to Aspire solutions afterwards and designed various static and dynamic web applications. He joined ERRA (a public sector organization which was developed to monitor the projects after the disastrous earthquake in Pakistan) as system analyst in August 2007 and supervised the data engineering group. He worked as Research Associate in NUST (National University of Science and Technology) for two years. He received his Masters in Information Technology (MS-IT) from NUST. He is currently a PhD student at the Graz University of Technology He is the author and coauthor of six papers and member of the software development team of Austria Forum.

Main research and project areas: information architecture, multimedia information systems, Web based learning; languages; artificial intelligence; information retrieval; Web 2.0; semantic Web; database.

PETRA KORICA-PEHSERL student of Software Development and Management at the Graz University of Technology (Austria) starting in 2003. Candidate for Ph.D. in Computer Science from the Graz University of Technology in 2014. Working for Microsoft in Vienna (Austria), Paris (France) and Zagreb (Croatia) since 2009.

She is the author of fifteen papers and member of the Editorial Board of Austria Forum. So far Petra has held more than 30 presentations at conferences worldwide.

Main research and project areas: Web based learning; languages; artificial intelligence; robotics; computer vision; information retrieval; Web 2.0; semantic Web; data; cloud computing. 
